\title{
Temperature and pressure constraints near the freezing point
}

\author{
Juan J. Morales and Marriaaia J. Nuevo \\ Departamento de Física, Facultad de Ciencias, Universidad de Extremadura, 06011 Badajoz, Spain \\ Luis F. Rull \\ Departamento de Física Teórica, Universidad de Sevilla, 41080 Sevilla, Spain \\ (Received 26 February 1990; revised manuscript received 3 May 1990)
}

\begin{abstract}
The isothermal-isobaric ensemble molecular-dynamics method $\operatorname{MD}(T, p, N)$ proposed by Nosé and Hoover is used to study the fluctuations in a two-dimensional Lennard-Jones fluid, close to the freezing point. The $T$ and $p$ constraints in this method do not affect the dynamical behavior of the system, since spontaneous fluctuations in the density allow the system to freeze and melt just as do the $T$ and $p$ fluctuations in the microcanonical ensemble $\operatorname{MD}(E, V, N)$ close to the melting zone.
\end{abstract}

\section{INTRODUCTION}

The dynamics of atomic systems with continuous interactions between the atoms requires numerical integration of Hamilton's equation of motion, ${ }^{1}$

$$
\begin{aligned}
& \frac{d H}{d \mathbf{p}_{i}}=\frac{d \mathbf{r}_{i}}{d t}=\dot{\mathbf{r}}_{i}=\frac{\mathbf{p}}{m}, \\
& \frac{d H}{d \mathbf{r}_{i}}=-\frac{d \mathbf{p}_{i}}{d t}=-\dot{\mathbf{p}}_{i}=\frac{d U(\mathbf{r})}{d \mathbf{r}_{i}},
\end{aligned}
$$

where $H=T(\mathbf{p})+U(\mathbf{r})$ is the Hamiltonian of the $N$-body system, $T(\mathbf{p})$ and $U(\mathbf{r})$ being the kinetic and potential energies, respectively. Newtonian mechanics implies that the energy and momentum are the conserved variables of the motion. In traditional molecular-dynamics (MD) experiments, the total energy $E$, the number of particles $N$, and the volume $V$ are conserved as the dynamics of the systems evolves in time. The time average of any property is an approximate measure of the microcanonical ensemble $\operatorname{MD}(E, V, N)$. For certain applications, it may be desirable to perform dynamical simulations at constant temperature and/or pressure leading to the canonical ensemble $\operatorname{MD}(T, V, N)$ and the isothermal-isobaric ensemble $\operatorname{MD}(T, p, N)$. However some of these techniques need the introduction of artificial processes in order to make this practical. Such schemes may be classified as nonNewtonian MD, since they are not based on Hamiltonians of motion for real systems, but use artifices of scaling that result in fictitious forces in order to satisfy some adopted definition for constant $T$ and $p .^{2}$ However, it is possible to show rigorously that certain of these methods give proper equilibrium ensemble averages.

Several methods have been developed to simulate the canonical ensemble, including the "stochastic" method ${ }^{3}$ in which collision with an imaginary heat-bath particle is taken into account, the "extended-system" method ${ }^{4}$ in which a degree of freedom is included which represents the reservoir in contact with the system, and the "constraints" method ${ }^{5}$ in which the velocities are rescaled at each time step by some factor in order to fix the kinetic energy of the system. Although other methods do not generate states in the canonical ensemble, they seem very useful for studying the changing state and systems reaching equilibrium at a different temperature. ${ }^{6}$ For the constant-pressure MD simulation, in all approaches it is inevitable that the system box must change volume as in constant-pressure Monte Carlo (MC) simulations. The nomenclature is the same as for the $\operatorname{MD}(T, V, N)$. The stochastic method ${ }^{7}$ is a MC calculation in which the Metropolis technique ${ }^{8}$ is applied to the randomly changed volume $\Delta V$. The extended method ${ }^{3}$ involves coupling the system to an external variable $V$, the volume of the system, where the coupling mimics the action of a piston on a real system. The constraints ${ }^{5}$ method makes the instantaneous pressure a constant of the motion. In two other particular methods, one allows the simulation box to change shape as well as size, ${ }^{9}$ and in the other the appropriate ensemble is not well identified. ${ }^{6}$

In a former study ${ }^{10}$ we presented some MD simulations for a system very close to the melting zone where fluctuations play an important role in the dynamic properties of the system. The simulations were performed using the traditional microcanonical ensemble, i.e., with no scaling procedure, and we found fluctuations for temperature and pressure which spontaneously make the system oscillate between two points of state. We conclude that simulations with any constraints method, such as the constant-temperature and/or constant-pressure methods, are incorrect near the melting zone because there are problems related to fluctuations with respect to space and time and the constraints can diminish the fluctuations or not allow them at all. Nevertheless, we now see this conclusion to be not completely general, because the system behavior depends on the method used and consequently on the way in which the constraints are applied.

Among the techniques reviewed here we chose the combination of the extended system method due to Nosé ${ }^{4}$ and the constraints method due to Hoover, ${ }^{11}$ leading to Nosé-Hoover (NH) formulation. ${ }^{12}$ There were two reasons for the choice: first, because the NH scaling procedure reproduces both the canonical and the isothermal-isobaric probability density in the phase space 
of a classical mechanical system, and second, because this method had been used previously to show that the dynamical behavior of the NH equations does not destroy the long-range bond correlation in the fluid near the freezing point. ${ }^{13}$ In the following we shall study the behavior of the temperature, pressure, and density fluctuations in the $\operatorname{MD}(T, p, N)$ ensemble in the two-dimensional (2D) system near the freezing point and the results will be compared with those obtained by $\operatorname{MD}(E, V, N)$ near the melting point. ${ }^{10}$

\section{SIMULATIONS AND RESULTS}

The equations of motion for the $\operatorname{MD}(T, p, N)$ ensemble in the NH formulation are ${ }^{13}$

$$
\frac{d \mathbf{x}_{i}}{d t}=\frac{\mathbf{p}_{i}}{m v^{1 / D}}
$$

and

$$
\frac{d \mathbf{p}_{i}}{d t}=\mathbf{f}_{i}-(\xi-\dot{\varepsilon}) \mathbf{p}_{i},
$$

where $\mathbf{x}_{i}=\mathbf{r}_{i} / V^{1 / D}$ are the reduced coordinates for the particle $i, D$ is the dimension of the space, $\mathbf{f}_{i}$ is the force, and $\xi$ and $\dot{\varepsilon}=\dot{V} / D V$ are the isothermal and isobaric friction coefficients which couple the system to a reservoir at $T=T_{\mathrm{ex}}$ and $p=p_{\mathrm{ex}}$, respectively.

The dynamical equations for the friction coefficients are

$$
\frac{d \xi}{d t}=\left[\sum_{i=1}^{N} \frac{p_{i}^{2}}{m}-D N k T\right) /\left(D N k T \tau_{T}\right)
$$

and

$$
\frac{d \dot{\varepsilon}}{d t}=\left(p-p_{\mathrm{ex}}\right) V /\left(k T \tau_{p}^{2}\right),
$$

where $\tau_{T}$ and $\tau_{p}$ are the isothermal and isobaric relaxation times of the system respectively which give the coupling strengths. Both relaxation times have a Gaussian distribution in the $\mathrm{NH}$ theory and they are related to the mean collision time. ${ }^{14,15}$

The NH isothermal-isobaric simulations were performed for a 2D Lennard-Jones system of $N=400$ particles with a cutoff distance of $r_{c}=2.5 \sigma$. The state point was set up at $k T / \varepsilon=0.7$ and $p \sigma^{2}=0.83$. This point is a fluid very close to the freezing point ${ }^{16}$ with a corresponding pressure of $p \sigma^{2} / \varepsilon=2.64$. These values for temperature and pressure were chosen as the fixed external $T$ and $p_{\text {ex }}$ of the reservoir in Eqs. (5) and (6). The other input values were $\tau_{T}=0.005$ and $\tau_{p}^{*}=\tau_{p} / \sqrt{N}=0.3$. $^{15}$ To integrate the equations of motion the Toxvaerd algorithm ${ }^{17}$ was used with a time step $h=0.005\left(m \sigma^{2} / \varepsilon\right)^{1 / 2}$, and the system was allowed to evolve in time about $120000 \mathrm{~h}$ taking averages values every $800 \mathrm{~h}$.

In Fig. 1 the fluctuation of temperature is plotted against the number of time steps for the $\operatorname{MD}(T, p, N)$ ensemble. The fluctuations are very small throughout the dynamic evolution of the system, in spite of the vicinity of the freezing zone where the fluctuations are large. This means that the isothermal friction coefficient works well at interchanging energy between system and reservoir through Eq. (5), diminishing the fluctuations around the fixed temperature as much as possible, as was to be expected in principle. The final mean value for the temperature was $k T / \varepsilon=0.7000 \pm 0.0007$. These fluctuations

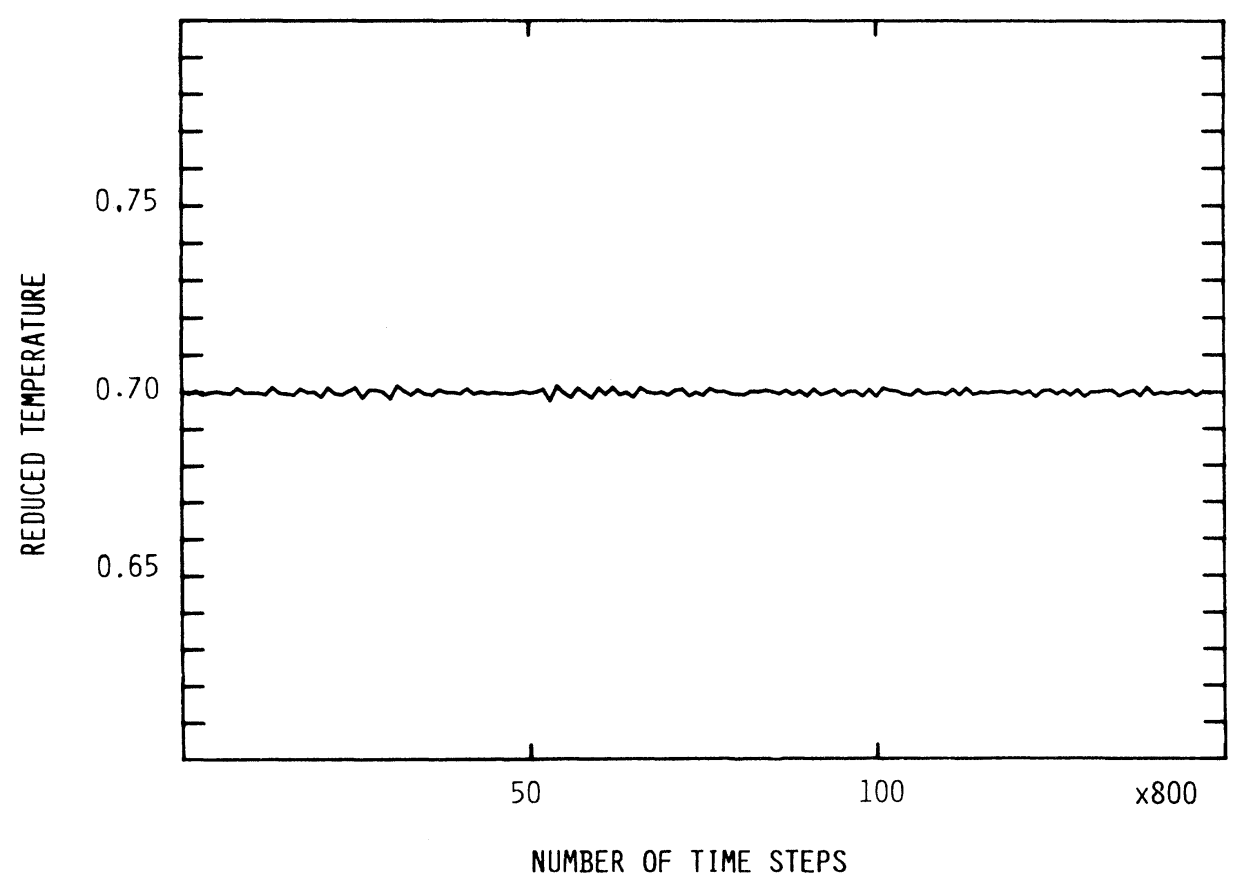

FIG. 1. Reduced-temperature fluctuations vs number of time steps for a two-dimensional fluid close to the freezing zone. 


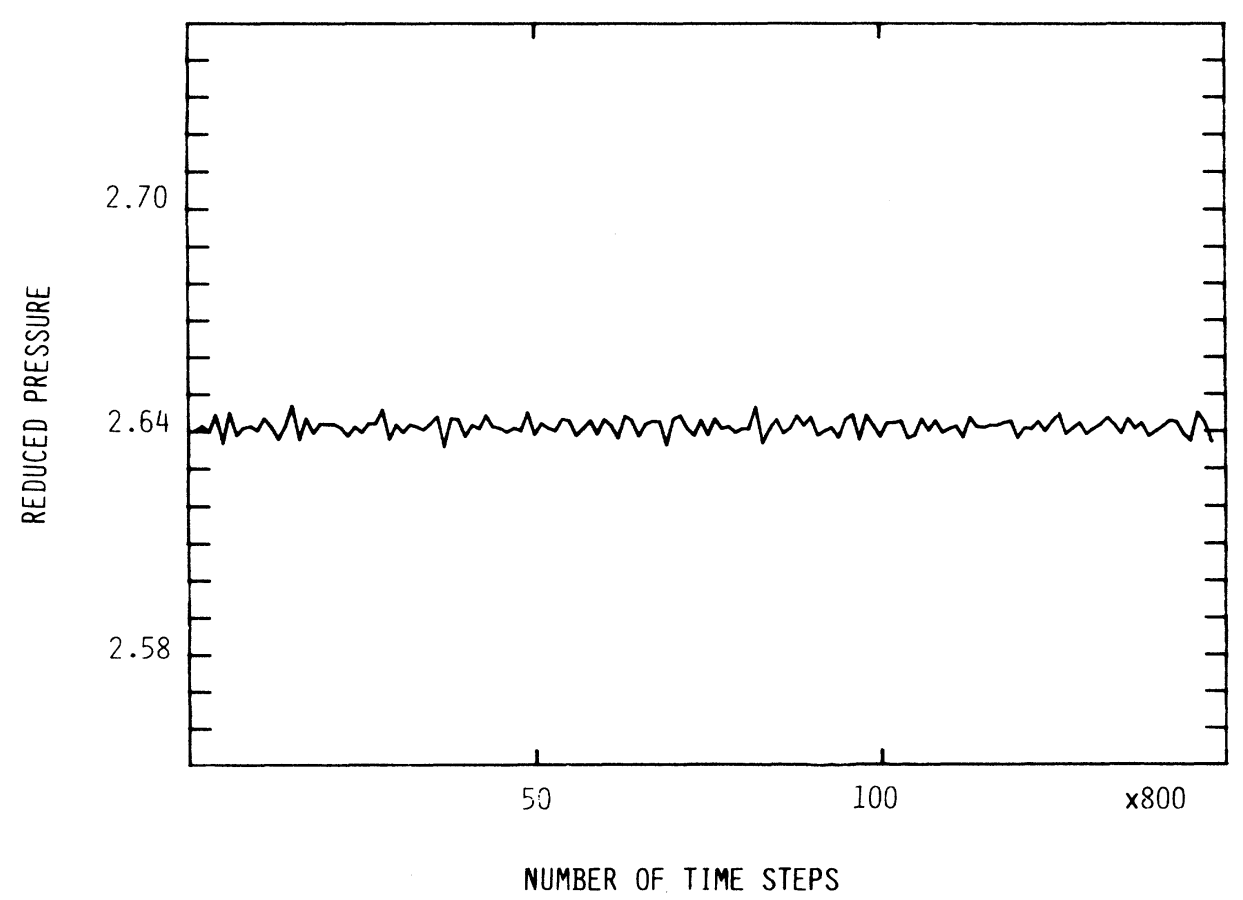

FIG. 2. As Fig. 1, but for the reduced pressure.

are about 14 times smaller than those for the corresponding $\operatorname{MD}(E, V, N)$ simulation in the melting zone. ${ }^{18}$

Similar behavior is found for the pressure fluctuations where the dynamical equation for the isobaric friction coefficient Eq. (6) maintains the system at constant pressure while allowing it to change its volume. These fluctuations look more regular that the temperature fluctuations and greater by about 3 times. The final mean value for the pressure was $p \sigma^{2} / \varepsilon=2.641 \pm 0.002$, with fluctuations 40 times smaller than the pressure fluctuation for the $\operatorname{MD}(E, V, N)$, as can be seen by comparison of the standard deviation and Fig. 2 with the results given in Ref. 18.

The time evolution of the density is very different, as Fig. 3 shows. The fluctuation is irregular and large due to the absence of constraints. On two occasions, after

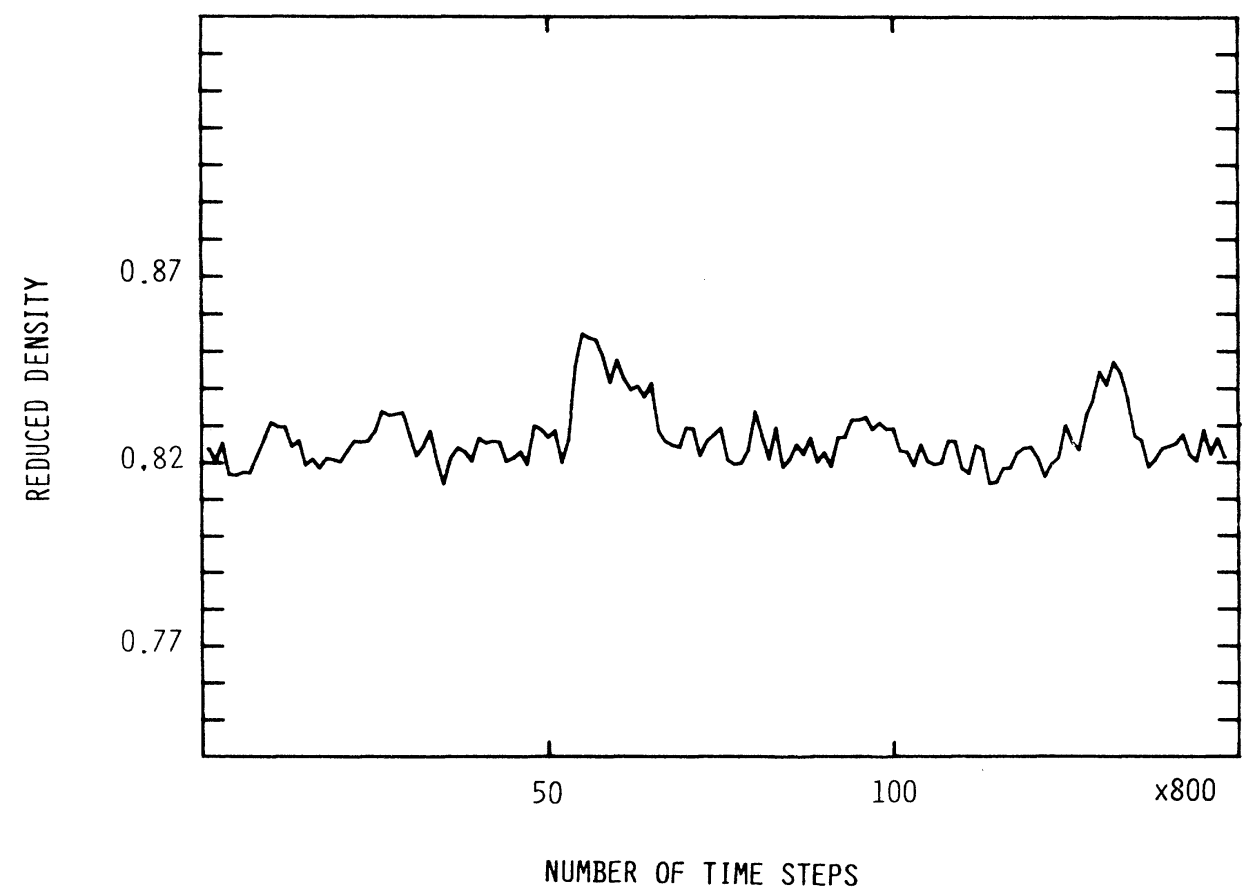

FIG. 3. As Fig. 1, but for the reduced density. 
about 44000 and 104000 time steps, fluctuations appear spontaneously which allow the system to reach densities within the freezing zone. ${ }^{16}$ The final mean value for the density was $p \sigma^{2}=0.827 \pm 0.010$, this standard deviation being the same as that corresponding to the temperature in the $\operatorname{MD}(E, V, N)$ ensemble at melting. These larger spontaneous fluctuations disappear when the system is far away from the freezing zone: simulation in the liquid system at $p \sigma^{2}=0.7606$ showed a standard deviation of \pm 0.0027 , fluctuations nearly four times smaller than before.

\section{DISCUSSION AND CONCLUSIONS}

If we compare these results $\operatorname{MD}(T, p, N)$ near the freezing point with those $\operatorname{MD}(E, V, N)$ corresponding to the solid near the melting zone, ${ }^{10}$ we can see that the two systems behave in the same way physically. The microcanonical ensemble allows temperature and pressure to fluctuate in the same way as the density in the isothermal-isobaric ensemble. Near a phase transition, both ensembles must undergo spontaneous fluctuations that drive the system back and forth between two points of state. The problem of the non-appearance of the fluctuations arises when the method used to simulate the $\operatorname{MD}(T, p, N)$ is incorrect, even though it yields time aver- ages for thermodynamically interesting variables to within the statistical accuracy of most experimental data. ${ }^{2}$ A very interesting discussion can be found in Refs. 7 and 16, where the validity of the stochastic method used for the volume change in Ref. 7 to investigate the melting transition is refuted in Ref. 16, since the error obtained with that method not only changes the correct location of the transition but also mistreats any density fluctuations in its vicinity.

We can therefore conclude that the constraints for temperature and pressure in the extended Nosé and Hoover method not only do not alter the natural evolution of the system, but also give the correct dynamical behavior including the zone of large fluctuations such as the freezing transition.

During the preparation of the present paper methods for implementing temperature and pressure controls at equilibrium and nonequilibrium in the $\mathrm{NH}$ dynamics have been published. ${ }^{19}$ These methods increase the efficiency of large-scale simulations but the detailed results are not yet available.

\section{ACKNOWLEDGMENT}

The cooperation of the University of Extremadura Computer Center is gratefully acknowledged.
${ }^{1}$ See, for example, H. Goldstein, Classical Mechanics (AddisonWesley, Reading MA, 1980), or M. P. Allen and D. J. Tildesley, Computer Simulation of Liquids (Clarendon, Oxford, 1989).

${ }^{2}$ F. F. Abraham, Adv. Phys. 35, 1 (1985).

${ }^{3}$ H. C. Andersen, J. Chem. Phys. 72, 2384 (1980).

${ }^{4}$ S. Nosé, Mol. Phys. 52, 255 (1984).

${ }^{5}$ D. J. Evans and G. P. Morris, Comput. Phys. Rep. 1, 297 (1984).

${ }^{6}$ H. J. C. Berendsen, J. P. M. Postma, W. F. Van Gusteren, A. Di Nola, and J. R. Haak, J. Chem. Phys. 81, 3684 (1984).

${ }^{7}$ S. W. Koch and F. F. Abraham, Phys. Rev. B 27, 2964 (1983).

${ }^{8}$ N. Metropolis, A. W. Rosenbluth, M. N. Rosenbluth, A. H. Teller, and E. Teller, J. Chem. Phys. 21, 1087 (1953).

${ }^{9}$ J. R. Ray, J. Chem. Phys. 79, 5128 (1983).
${ }^{10}$ L. F. Rull, J. J. Morales, and F. Cuadros, Phys. Rev. B 32, 6050 (1985).

${ }^{11}$ W. G. Hoover, Phys. Rev. A 31, 1695 (1985).

${ }^{12}$ S. Nosé, Mol. Phys. 57, 187 (1986).

${ }^{13}$ J. J. Morales, S. Toxvaerd, and L. F. Rull, Phys. Rev. A 34, 1495 (1986).

${ }^{14}$ D. J. Evans and B. L. Holian, J. Chem. Phys. 83, 4069 (1985).

${ }^{15}$ L. F. Rull, J. J. Morales, and S. Toxvaerd, Phys. Rev. A 38, 4309 (1988).

${ }^{16}$ S. Toxvaerd, Phys. Rev. B 29, 2821 (1984).

${ }^{17}$ S. Toxvaerd, J. Comput. Phys. 47, 444 (1982).

${ }^{18}$ J. J. Morales, F. Cuadros, and L. F. Rull, J. Chem. Phys. 86, 2960 (1987).

${ }^{19}$ B. L. Holian, A. J. De Groot, W. G. Hoover, and C. G. Hoover, Phys. Rev. A 41, 4552 (1990). 